This is an electronic reprint of the original article. This reprint may differ from the original in pagination and typographic detail.

Author(s): Renk, Thorsten

Title: $\quad$ Sensitivity of jet quenching to enhancement of the medium opacity near TC

Year: $\quad 2014$

Version:

Please cite the original version:

Renk, T. (2014). Sensitivity of jet quenching to enhancement of the medium opacity near TC. Physical Review C, 89(June), Article 067901.

https://doi.org/10.1103/PhysRevC.89.067901

All material supplied via JYX is protected by copyright and other intellectual property rights, and duplication or sale of all or part of any of the repository collections is not permitted, except that material may be duplicated by you for your research use or educational purposes in electronic or print form. You must obtain permission for any other use. Electronic or print copies may not be offered, whether for sale or otherwise to anyone who is not an authorised user. 


\title{
Sensitivity of jet quenching to enhancement of the medium opacity near $T_{C}$
}

\author{
Thorsten Renk ${ }^{*}$ \\ Department of Physics, P.O. Box 35, FI-40014 University of Jyväskylä, Finland \\ and Helsinki Institute of Physics, P.O. Box 64, FI-00014 University of Helsinki, Finland \\ (Received 28 February 2014; revised manuscript received 14 April 2014; published 13 June 2014)
}

\begin{abstract}
One of the main goals of the study of high transverse momentum $\left(P_{T}\right)$ observables in the context of ultrarelativistic heavy-ion collisions is the determination of properties of QCD matter. In particular, the transport coefficients $\hat{q}$ and $\hat{e}$ characterizing the interaction of the medium with a high $p_{T}$ parton are accessible via hard probes. However, a precision extraction of their temperature dependence from current data faces the problem that neither the space-time geometry of the evolving matter nor the link between thermodynamics and transport coefficients is unambiguously known. Thus, various conjectured scenarios exist for how thermodynamics and transport coefficients behave close to the phase transition. While often a behavior with the energy density $\hat{q} \sim \epsilon^{3 / 4}$ is assumed, leading to a decrease of the scaled $\hat{q}(T) / T^{3}$ close to the critical temperature $T_{C}$, other scenarios expect instead a near $T_{C}$ enhancement of jet quenching. In this work, the response of both the extraction of $\hat{q}$ and the enhancement of $v_{2}$ at high $P_{T}$ to modification of jet quenching close to $T_{C}$ is investigated within YaJEM, a well-tested in-medium shower evolution Monte Carlo code, embedded into a fluid dynamics simulation for the medium, thus allowing a gauge of the magnitude of the effect in a realistic framework.
\end{abstract}

PACS number(s): $25.75 . \mathrm{Gz}$

Introduction. High $P_{T}$ observables are a cornerstone of the ultrarelativistic heavy-ion $(A A)$ program at both the BNL Relativistic Heavy Ion Collider (RHIC) and the CERN Large Hadron Collider (LHC). One key goal of this program is the extraction of properties of the produced QCD matter droplet, for instance, in terms of transport coefficients and their temperature dependence. Two such coefficients, $\hat{q}$ (the mean gain in transverse momentum squared of a high $p_{T}$ parton per unit path length) and $\hat{e}$ (the mean longitudinal momentum loss of a high $p_{T}$ parton per unit path length) are particularly relevant in this context. Here, $\hat{q}$ is responsible for energy loss from hard partons into medium-induced soft gluon radiation whereas $\hat{e}$ causes energy loss into nonperturbative medium degrees of freedom as well as some hard recoil of medium constituents (see, e.g., [1] for a review). The only known way to extract the temperature dependence of these coefficients from the data is to use perturbative QCD (pQCD) to compute the primary hard parton production and then to embed a model for the parton-medium final state interaction into a fluid-dynamical simulation of the matter to obtain the medium modification to the final state. The transport coefficient is in this approach computed as a function of thermodynamical parameters used in the fluid dynamics, e.g., energy density $\epsilon$, temperature $T$, or entropy density $s$ as well as potentially the flow vector $u^{\mu}$ of the medium relative to the c.m. frame of the collision.

Based on the notion of counting the number density of potential scattering centers in an ideal gas, many jet quenching models assume

$$
\hat{q} \sim T^{3}, \quad \hat{q} \sim s, \quad \text { or } \quad \hat{q} \sim \epsilon^{3 / 4},
$$

which for an ideal gas equation of state $\epsilon=3 p$ all coincide. Differences to the ideal gas for all three expressions occur

*thorsten.i.renk@jyu.fi close to the phase transition and in the hadronic phase and have previously been discussed in, e.g., [2]. In the presence of a finite flow value, a relativistic correction term dependent on the local flow rapidity $\rho$ at the position $\zeta$ and the angle $\alpha$ between the parton momentum vector and flow vector has been found in both weak and strong couplings as [3,4]

$$
F(\rho(\zeta), \alpha(\zeta))=\cosh \rho(\zeta)-\sinh \rho(\zeta) \cos \alpha(\zeta)
$$

In practice, this factor corresponds to a small correction [5]. Given such a setting, a $\chi^{2}$ fit of the proportionality constant between the transport coefficient and thermodynamical parameter to, e.g., the single inclusive hadron nuclear suppression factor $R_{A A}$ is possible [6] and results in a temperature dependence of $\hat{q}$ compatible with the data. However, such a procedure does not yield consistent results across different models [7], indicating that the uncertainties related to the choice of the model are substantial. This in turn is related to the fact that $R_{A A}$ in itself is not sufficient to falsify models reliably. One solution is thus to include other observables into the fit, for instance, the suppression factor of the back-to-back coincidences $I_{A A}$ [8]; however, for multiple observables and across the full parameter space of available models, such a strategy soon becomes prohibitively expensive.

A further source of uncertainty is that any temperature dependence thus extracted is not determined in a data-driven way but assumed a priori using a relation like Eq. (1). However, in [9-12] a scenario was suggested in which parton-medium interaction is not reduced but parametrically enhanced close to the phase transition temperature $T_{C}$ ("near $T_{C}$ enhancement," referred to as NTC in the following). This suggestion was driven by the need to explain the experimentally observed large split between in-plane and out-of-plane particle emission at high $P_{T}$ [13] when the dependence of $R_{A A}$ with respect to the angle with the event plane $\phi$ is considered in noncentral $A A$ collisions. A systematic study across different models [14] has, however, demonstrated that the magnitude of the split 
is influenced by many factors, among them the path-length dependence of energy loss, the initial eccentricity of the medium, the viscous entropy production, and the total size of the space-time volume in which hard partons interact with the medium. Taking all these uncertainties into account, it is not clear whether there is a remaining tension with the data; however, there is a trend across several models to underpredict the spread [15-17]. So far, NTC has not been tested using a realistic model of jet quenching, but rather a simple parametrization. The aim of this work is to quantify the potential effect of a NTC scenario on both the extraction of a transport coefficient $\hat{q}$ and the spread between in-plane and out-of-plane $R_{A A}$ in a well-tested state of the art jet quenching model and to verify whether the order of magnitude of the effect obtained in $[9,10]$ persists.

The observable. The observable considered in this study is the single inclusive hadron suppression factor $R_{A A}$ which is defined as the yield of high $P_{T}$ hadrons from an $A A$ collision normalized to the yield in $p-p$ collisions at the same energy corrected for the number of binary collisions,

$$
R_{A A}\left(p_{T}, y\right)=\frac{d N_{A A}^{h} / d p_{T} d y}{T_{A A}(\mathbf{b}) d \sigma^{p p} / d p_{T} d y} .
$$

The default expectation is $R_{A A}<1$ in the medium since parton-medium interaction is expected to lead to a flow of high $p_{T}$ parton momentum into medium degrees of freedom, thus effectively suppressing the yield in any given momentum bin. Nuclear initial state effects or fluctuations in elastic energy loss can, however, cause $R_{A A}>1$ in some kinematical regions.

Experimentally, $R_{A A}$ can readily be obtained with respect to the angle $\phi$ of a hard hadron with the bulk matter $v_{n}$ event plane orientation $\phi_{2}$ where $v_{n}$ is the $n$th coefficient in a harmonic expansion

$$
\frac{d N}{d \phi}=\frac{N}{2 \pi}\left(1+\sum_{n}\left[2 v_{n} \cos (n \phi)\right]\right)
$$

of the angular distribution of the bulk particle yield $d N / d \phi$. The spread $S_{\text {out }}^{\text {in }}=R_{A A}(0)-R_{A A}(\pi / 2)$ between in-plane and out-of-plane emission is an important observable sensitive to both the medium geometry and the energy loss path-length dependence.

Knowledge of $R_{A A}(0)=R_{A A}^{\text {in }}$ and $R_{A A}(\pi / 2)=R_{A A}^{\text {out }}$ is approximately equivalent to the angular averaged $R_{A A}$ and the second harmonic coefficient $v_{2}$ at high $P_{T}$, since if the modulation is a pure second harmonic, then

$$
R_{A A}^{\text {in }}=R_{A A}\left(1+2 v_{2}\right) \quad \text { and } \quad R_{A A}^{\text {out }}=R_{A A}\left(1-2 v_{2}\right),
$$

with $R_{A A}$ the angular averaged value. Colloquially $v_{2}$ is frequently referred to as an elliptic flow coefficient, but this is highly misleading at high $P_{T}$ because the angular modulation is not driven by any flow phenomenon but by the different strength of the parton-medium interaction dependent on the density and length of traversed matter. The attenuation is known to be a nonlinear function of the length; in particular, no matter how strong the interaction with the medium, $R_{A A}>0$ is always true. This implies that for sufficiently low values of the average $R_{A A}$ and high $v_{2}$, Eq. (4) cannot be fulfilled and saturation leads to a distortion of the resulting angular structure from a pure $v_{2}$ modulation even if the matter distribution has a perfect second harmonic spatial eccentricity $\epsilon_{2}$. At the minimum of $R_{A A}$ at LHC at about $P_{T}=10 \mathrm{GeV}$, this creates a spurious $v_{4} \approx 0.2 v_{2}$ for the model used in this study. Since this is commonly done in the literature, we will in the following discuss $\phi$ dependent physics nevertheless in terms of $v_{2}$ with the above caveats in mind.

Extraction of $\hat{q}$. In the following, we parametrize NTC by the expression

$$
\hat{q}(T)=2 K T^{3}\left[1+c \exp \left(-\frac{\left(T-T_{C}\right)^{2}}{\sigma^{2}}\right)\right] F(\rho, \alpha),
$$

with $F(\rho, \alpha)$ as in Eq. (2), $K$ a free parameter regulating the overall strength of the parton-medium interaction, and $c, \sigma$ characterizing the strength and region of influence of the NTC (currently no fundamental theory constrains these values). To gain some insight into how NTC parameters map into observables, we test $\sigma=10 \mathrm{MeV}$ and $\sigma=30 \mathrm{MeV}$ in the following as well as $c$ in the range from 0 to 3 .

Equation (5) is applied to a $2+1 \mathrm{~d}$ fluid dynamical simulation of the bulk matter evolution for $2.76 \mathrm{ATeV} \mathrm{Pb}-\mathrm{Pb}$ collisions [18]. Using the local temperature $T$, the transport coefficient for every space-time point dependent on the specific hard parton trajectory through the matter can be obtained. We generate a distribution of hard partons based on leading order perturbative QCD expressions in a Monte Carlo routine and initialize them in the transverse plane based on the binary collision probability distribution with a specified orientation with respect to the bulk $v_{2}$ event plane.

Parton-medium interaction is computed using the scenario YaJEM-DE [19] of the in-medium shower evolution code YaJEM [20] which is an extension of the PYSHOW routine [21] simulating the QCD scale evolution in a vacuum. The reader interested in details of the simulation is referred to these works. YaJEM-DE is well tested against a number of different high $P_{T}$ observables both at RHIC and LHC, among them also observables with multiparticle final states, e.g., the dijet imbalance [22] and jet-hadron [23] correlations. However, it should be noted that this is perhaps not central to the present work where we are interested in the relative change of $\hat{q}$ and $v_{2}$ due to NTC rather than absolute values. In comparing the results of a systematic investigation of jet quenching using different hydrodynamical backgrounds [14], an empirical observation is that, at least for reasonably realistic models, the relative change in high $P_{T} v_{2}$ driven by properties of the fluid dynamics is with an accuracy better than $10 \%$ independent of the details of the parton-medium interaction model used. The absolute value of $v_{2}$, however, shows a strong dependence on both fluid dynamics and jet quenching model.

$\hat{q}(T)$ is determined for any selection of $c$ and $\sigma$ by fitting $K$ in Eq. (5) to the angular averaged $R_{A A}$ in $0-10 \%$ central collisions to ALICE charged hadron data [24] at $P_{T}=$ $10 \mathrm{GeV}$. Figure 1 shows the curves of $\hat{q} / T^{3}$ resulting from these fits for the various scenarios in comparison with the default ansatz $\hat{q} \sim \epsilon^{3 / 4} F(\rho, \alpha)$, assuming that jets decouple at the $T_{F}=0.13 \mathrm{GeV}$ hypersurface. From the results, it becomes clear that the high $T$ determination of $\hat{q}$ has no strong uncertainty associated with the near- $T_{C}$ behavior of quenching. All 


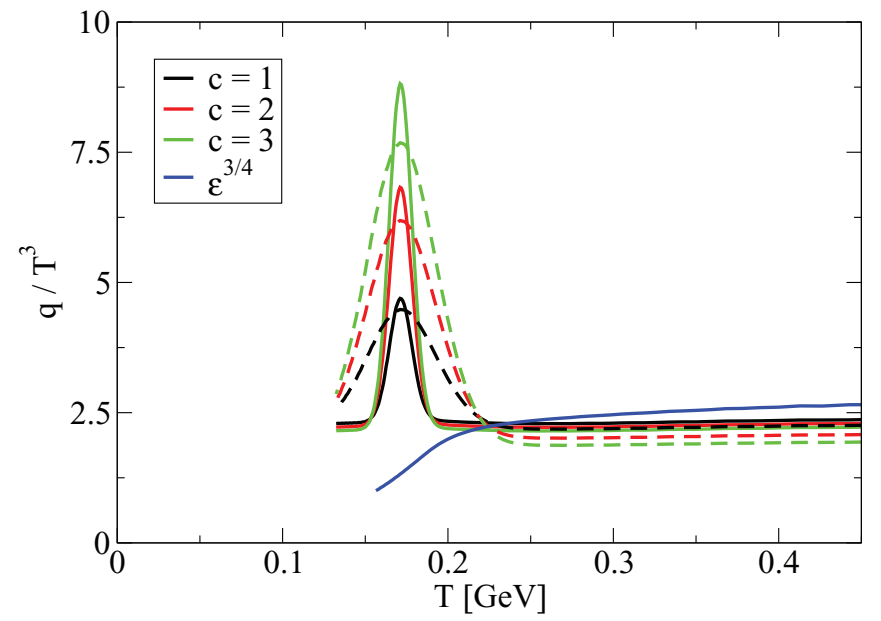

FIG. 1. (Color online) Temperature dependence of the scaled transport coefficient $\hat{q} / T^{3}$ for various near $T_{C}$ enhancement scenarios (see text) as determined by fits to $R_{A A}$ in $2.76 A \mathrm{TeV} 0-10 \%$ central $\mathrm{Pb}-\mathrm{Pb}$ collisions at $P_{T}=10 \mathrm{GeV}$. Shown are $\sigma=10 \mathrm{MeV}$ (solid) and $\sigma=30 \mathrm{MeV}$ (dashed).

scenarios find a stable value of $\hat{q}(T) / T^{3} \sim 2.4$ to $2.5 \mathrm{GeV}$. This value is well in line with other model results $[16,25]$. Turning the argument around, one finds that as expected the near $T_{C}$ dynamics is not well constrained by fitting angular averaged $R_{A A}$. The main uncertainty for a reliable determination of $\hat{q}$ still comes from the details of the fluid dynamical evolution [26]. For illustration, assuming that hard partons decouple at a temperature of $T_{F}=0.16 \mathrm{GeV}$, the qualitative picture stays the same, but instead $\hat{q}(T) / T^{3} \sim 3.8$ to 4.1 is found at high $T$ for the various scenarios. One can conclude that precise matter geometry and jet-medium decoupling are currently the largest uncertainty for a determination of $\hat{q}$.

Impact on $v_{2}$. To assess the importance of NTC for the magnitude of $v_{2}$ at high $P_{T}$, we leave $\hat{q}(T)$ as determined by the mean $R_{A A}$ in central collisions as described above and use the same fluid dynamics computation for 30-40\% centrality. At $P_{T}=10 \mathrm{GeV}$, we compute $R_{A A}(\phi)$ and fit the expression

$$
R_{A A}(\phi)=\left\langle R_{A A}\right\rangle\left[1+2 v_{2} \cos (2 \phi)\right]
$$

to the result. As discussed above, this is not a perfect fit because there is a spurious $v_{4}$ modulation. Nevertheless, for this work we only focus on the $v_{2}$ coefficient.

We repeat this procedure for every NTC scenario and plot the relative enhancement over the default assumption $\hat{q} \sim \epsilon^{3 / 4}$. Note again that only the relative enhancement is meaningful at this stage - the absolute value of $v_{2}$ depends on multiple factors, among them the path-length dependence of the jet-medium interaction and the importance of quantum coherence effects, the initial eccentricity distribution of the matter, the amount of viscous entropy production dependent on the value of viscosity over entropy density $\eta / s$, or the precise choice of the decoupling surface for jets from the medium [14]. By considering the relative enhancement only, many of these uncertainties approximately drop out.

The result is shown in Fig. 2. A few observations can readily be made: First, for all scenarios tested, the highest

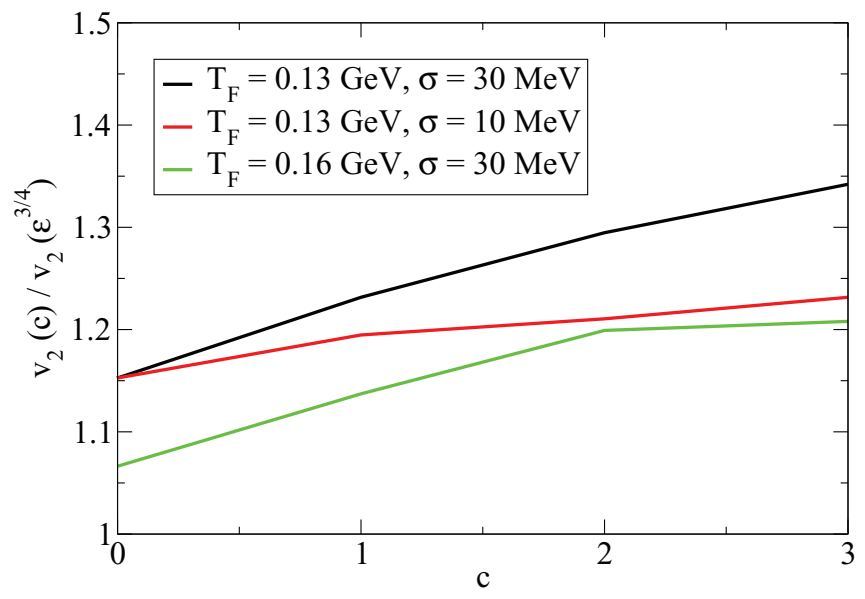

FIG. 2. (Color online) Relative increase of $v_{2}$ at $P_{T}=10 \mathrm{GeV}$ for $30-40 \%$ central $\mathrm{Pb}-\mathrm{Pb}$ collisions at $2.76 \mathrm{~A} \mathrm{TeV}$ for the various scenarios of NTC (see text) relative to the default $\epsilon^{3 / 4}$ scenario as a function of the NTC strength parameter $c$.

enhancement found is $35 \%$. This is sizable and comparable with, e.g., the combined effect of slow thermalization and viscous entropy production [14], but smaller than the influence of the space-time extent of the medium. For weak coupling scenarios [15-17] which tend to underpredict $v_{2}$ at high $P_{T}$, NTC is favored but cannot unambiguously be identified as the one dominating factor. Second, about half of the possible effect already results from not having a reduction of quenching around $T_{C}$, larger values of $c$ corresponding to more pronounced enhancement still increase $v_{2}$, but there are indications for a saturation. Third, and perhaps not surprisingly, the effect of NTC is more pronounced the more NTC is probed by the hard parton. Both when the evolution is carried to a lower decoupling temperature and when the region in which NTC is effective is increased, a higher relative enhancement of $v_{2}$ is found. Comparing with the results obtained previously in [11] using a simple parametrized energy loss model, the enhancement of about $20 \%$ of a NTC scenario over a constant scenario is of the same order of magnitude.

Discussion. In this work, the effect of a near $T_{C}$ enhancement of the parton-medium interaction on both the extraction of the transport coefficient $\hat{q}$ in central collisions and the enhancement of $v_{2}$ in noncentral collisions has been investigated. The results have for the first time been obtained in a well-constrained and realistic model combination of fluid dynamics and parton-medium interactions and agree with the order of magnitude obtained before using a parametrized energy loss [11]. Given the empirically observed independence of the relative response of $v_{2}$ to changes in the fluid dynamics from details of the energy loss model [14], this suggests that the size of the NTC effect is fairly robust against changes in background geometry or energy loss model.

It was found that the extraction of $\hat{q}(T) / T^{3}$ from $R_{A A}$ in central collisions in the region $T>250 \mathrm{MeV}$, i.e. where the quark-gluon plasma (QGP) is expected to exist, is not substantially influenced by the near $T_{C}$ region. This is fortunate, as it allows one to access the physics of the QGP without 
a full understanding of the phase transition and hadronization. However, other factors, for instance, the uncertainty in the total size of the region in which parton and medium interact, still pose a challenge for any precision extraction.

In contrast, $v_{2}$ was found to be sensitive to NTC as suggested in $[9,10,12]$ on a level of a $~ 35 \%$ enhancement, confirming the previous results in a realistic and well-tested setup. This is in line with results from [14] that $v_{2}$ can generically be expected to increase when energy loss from the leading parton happens later. Comparing weak coupling scenarios with data, such an increase is certainly supported and indicates that NTC is favored over a reduction of the interaction near $T_{C}$. However, given the sizable other systematic uncertainties affecting the absolute value of $v_{2}$, it is difficult to tell whether NTC is required by the data and to unambiguously determine the size of the enhancement. An answer to this question will require a systematic picture across several different high $P_{T}$ observables and will be the topic of a future investigation.

Acknowledgments. This work is supported by the Academy researcher program of the Academy of Finland, Project No. 130472.
[1] A. Majumder and M. Van Leeuwen, Prog. Part. Nucl. Phys. A 66, 41 (2011).

[2] S. A. Bass, C. Gale, A. Majumder, C. Nonaka, G.-Y. Qin, T. Renk, and J. Ruppert, Phys. Rev. C 79, 024901 (2009).

[3] R. Baier, A. H. Mueller, and D. Schiff, Phys. Lett. B 649, 147 (2007).

[4] H. Liu, K. Rajagopal, and U. A. Wiedemann, J. High Energy Phys. 03 (2007) 066.

[5] T. Renk, J. Ruppert, C. Nonaka, and S. A. Bass, Phys. Rev. C 75, 031902 (2007).

[6] A. Adare et al. (PHENIX Collaboration), Phys. Rev. Lett. 101, 232301 (2008).

[7] N. Armesto, B. Cole, C. Gale, W. A. Horowitz, P. Jacobs, S. Jeon, M. van Leeuwen and A. Majumder et al., Phys. Rev. C 86, 064904 (2012).

[8] N. Armesto, M. Cacciari, T. Hirano, J. L. Nagle, and C. A. Salgado, J. Phys. G 37, 025104 (2010).

[9] J. Liao and E. Shuryak, Phys. Rev. Lett. 102, 202302 (2009).

[10] X. Zhang and J. Liao, Phys. Rev. C 87, 044910 (2013).

[11] X. Zhang and J. Liao, Phys. Rev. C 89, 014907 (2014).
[12] D. Li, J. Liao, and M. Huang, arXiv:1401.2035.

[13] S. Afanasiev et al. (PHENIX Collaboration), Phys. Rev. C 80, 054907 (2009).

[14] T. Renk, H. Holopainen, U. Heinz, and C. Shen, Phys. Rev. C 83, 014910 (2011).

[15] D. L. Winter, J. Phys. Conf. Ser. 230, 012017 (2010).

[16] J. Xu, A. Buzzatti, and M. Gyulassy, arXiv:1402.2956.

[17] T. Renk, Phys. Rev. C 83, 024908 (2011).

[18] T. Renk, H. Holopainen, R. Paatelainen, and K. J. Eskola, Phys. Rev. C 84, 014906 (2011).

[19] T. Renk, Phys. Rev. C 84, 067902 (2011).

[20] T. Renk, Phys. Rev. C 79, 054906 (2009).

[21] M. Bengtsson and T. Sjöstrand, Phys. Lett. B 185, 435 (1987); Nucl. Phys. B 289, 810 (1987); E. Norrbin and T. Sjöstrand, ibid. 603, 297 (2001).

[22] T. Renk, Phys. Rev. C 86, 061901 (2012).

[23] T. Renk, Phys. Rev. C 87, 024905 (2013).

[24] K. Aamodt et al. (ALICE Collaboration), Phys. Lett. B 696, 30 (2011).

[25] Xin-Nian Wang, talk Hard Probes 2013.

[26] T. Renk, Phys. Rev. C 85, 044903 (2012). 